\title{
Resonance Self-shielding Calculation for Plate-type Fuel Assemblies
}

\author{
Fan Xia, Hongchun Wu, Yunzhao Li", Jiewei Yang \\ School of Nuclear Science and Technology, Xi'an Jiaotong University, \\ 28 West Xianning Road, Xi'an,Shaanxi 710049, China \\ *yunzhao@xjtu.edu.cn
}

\begin{abstract}
Owing to its ability to handle arbitrary geometry and high computation efficiency, subgroup method is a widely used resonance self-shielding method. Ordinarily, the subgroup parameters are generated from homogenous resonance integral tables, and then can be used to calculate heterogeneous problems via the equivalence theory. However, it cannot provide accurate results especially for the plate-type assemblies with strong heterogeneity. What's more, the fuel enrichment in plate-type assemblies is relatively much higher than the common rod-type ones. As a result, the resonance interference effect in plate-type fuels is particularly intense. To solve this problem and to provide accurate effective self-shielded cross-sections for plate-type fuel assemblies, 1-D plate problems are used to generate subgroup parameters. In order to deal with resonance interference, the resonance interference factors are generated by equivalent homogenous problems solved by 0 -D hyperfine group solver. To avoid the computational burden caused by too many hyperfine group calculations, the importance of resonance isotopes is calculated in advance to select important resonance isotopes. Important and non-important ones are handled by different ways respectively. JRR-3M plate-type assemblies are used to test the newly method. Numerical results show that the relative errors of effective self-shielded crosssections are generally less than $1.5 \%$ compared with the reference.
\end{abstract}

KEYWORDS: plate-type assembly, subgroup method, resonance interference factors

\section{INTRODUCTION}

Plate-type fuel is very common in research reactors which are small in size and complex in geometry compared with commercial PWRs. Resonance calculation is essential to deterministic neutron transport calculation, while most of the resonance calculation methods are limited to regular geometry.

Owing to its ability to handle arbitrary geometry and high computation efficiency, subgroup method [1] is a good choice for plate-type fuel assembly calculation. Subgroup parameters are generated in advance from the homogenous resonance integral tables. However, researches indicate that the scheme with homogenous resonance integral tables cannot provide accurate results to heterogeneous problems via the equivalence theory. Thus, problem-dependent heterogeneous resonance integral tables should be used instead [2,3]. HELIOS code firstly applied heterogeneous RI tables to subgroup method [4]. A subgroup weight generation method was also proposed based on shielded pin cell cross-section conservation [5]. 
Another problem leading to the poor accuracy of resonance calculation is the resonance interference effect. Ordinarily, the fuel enrichment in plate-type assemblies is relatively higher than the common rodtype ones, greater than $10 \%$. It makes the resonance interference between different resonance isotopes intense. As the fuel burns, the number of resonance isotopes can be greatly increased and the problem becomes more complex.

In this paper, the subgroup parameters are generated from series of 1-D plate problems which are solved by 1-D hyperfine group code. To treat the resonance interference, the resonance interference factors [6] are calculated by equivalent homogenous problems solved by 0 -D hyperfine group solver to correct the self-shielded cross-sections. The importance of resonance isotopes is introduced in our theory to classify resonance isotopes which will be treated by different ways to reduce the computational burden. Additionally, the JRR-3M [7] plate-type fuel assemblies are calculated by the method developed.

\section{METHODOLOGIES}

In this section, the subgroup parameters generation method based on 1-D plate is introduced firstly. The problems of negative subgroup parameters and numerical instability are not discussed here. All of these problems and their solutions are explained in detail in our previous work [8]. The method of treating resonance interference is illustrated in the last half part of this section.

\subsection{Subgroup parameters generation based on 1-D plate}

Subgroup method divides each energy group into several subgroups according to the magnitude of crosssections. The energy in each subgroup is not continuous. The self-shielded cross-section in a group can be written as:

$$
\sigma_{x, g}=\frac{\sum_{n} \sigma_{x, g, n} \phi_{g, n} w_{x, g, n}}{\sum_{n} \phi_{g, n} w_{x, g, n}}
$$

where $n$ donates the serial number of subgroup; the subgroup level $\sigma_{x, g, n}$ and the subgroup weight $w_{x, g, n}$ are called subgroup parameters; $\phi_{g, n}$ is the subgroup flux obtained by solving the subgroup fixed source equation Eq.(2).

$$
\nabla \cdot \Omega \varphi_{g, n}(r, \Omega)+\left(\Sigma_{a, g, n}(r)+\lambda \Sigma_{p}(r)\right) \varphi_{g, n}(r, \Omega)=\frac{1}{4 \pi} \lambda \Sigma_{p}(r)
$$

where $\Sigma_{a, g, n}$ is the absorption cross-section in subgroup $n ; \lambda$ is the intermediate resonance factor; $\Sigma_{p}$ is the potential scattering cross-section.

For a homogeneous mixture, the scalar flux of subgroup $n$ can be expressed as:

$$
\phi_{g, n}=\frac{\lambda \Sigma_{p}}{\Sigma_{a, g, n}+\lambda \Sigma_{p}}=\frac{\sigma_{b}}{\sigma_{a, g, n}+\sigma_{b}}
$$


$\sigma_{b}$ is the background cross-section defined as:

$$
\sigma_{b}=\frac{\lambda \Sigma_{p}}{N_{r}}
$$

where $N_{r}$ is the number density of the resonance isotope. Thus the shielded cross-section for a homogeneous problem can be written as follows by taking Eq.(3) into Eq.(1).

$$
\sigma_{x, g}=\frac{\sum_{n} \sigma_{x, g, n} \frac{\sigma_{b}}{\sigma_{a, g, n}+\sigma_{b}} w_{g, n}}{\sum_{n} \frac{\sigma_{b}}{\sigma_{a, g, n}+\sigma_{b}} w_{g, n}}
$$

While for a heterogeneous problem, since there is leakage, the background cross-section should be expressed as follows according to the equivalence theory.

$$
\sigma_{b}=\frac{\lambda \Sigma_{p}}{N_{r}}+\frac{\Sigma_{e, n}}{N_{r}}
$$

where $\Sigma_{e, n}$ is the escape cross-section which is associated with the leakage effect. Using Eq.(3) and Eq.(6), the escape cross-section can be expressed as follows:

$$
\Sigma_{e, n}=\frac{\Sigma_{a, g, n} \phi_{g, n}}{1-\phi_{g, n}}-\lambda \Sigma_{p}
$$

In heterogeneous problems, the escape cross-sections and background cross-sections become dependent on the subgroup levels. Then, the self-shielded cross-section for a heterogeneous problem can be written as:

$$
\sigma_{x, g}=\frac{\sum_{n} \sigma_{x, g, n} \frac{\sigma_{b, n}}{\sigma_{a, g, n}+\sigma_{b, n}} w_{g, n}}{\sum_{n} \frac{\sigma_{b, n}}{\sigma_{a, g, n}+\sigma_{b, n}} w_{g, n}}
$$

Based on Eq.(8), the least-square problem aimed at fitting subgroup parameters can be established as follows: 


$$
\min F(\boldsymbol{\sigma}, \mathbf{w})=\sum_{k=1}^{K}\left[1-\frac{\sum_{n=1}^{N} \sigma_{g, n} \frac{\sigma_{g, b n, k}\left(\sigma_{g, n}\right)}{\sigma_{g, n}+\sigma_{b n, k}\left(\sigma_{g, n}\right)} w_{g, n}}{\sigma_{g, k}^{\operatorname{Ref}} \sum_{n=1}^{N} \frac{\sigma_{g, b n, k}\left(\sigma_{g, n}\right)}{\sigma_{g, n}+\sigma_{g, b n, k}\left(\sigma_{g, n}\right)} w_{g, n}}\right]^{2}
$$

where vector $\boldsymbol{\sigma}=\left(\sigma_{1}, \ldots, \sigma_{N}\right)^{T}$ and $\mathbf{w}=\left(w_{1}, \ldots, w_{N}\right)^{T} ; K$ is the number of heterogeneous dilution cases; $\sigma_{g, k}^{\text {Ref }}$ is the reference self-shielded cross-sections of the heterogeneous problems calculated by solving continuous energy slowing-down equation using Monte Carlo code or hyperfine group code.

The background cross-sections $\sigma_{g, b n, k}$ and subgroup levels $\sigma_{g, n}$ are interacted on each other, so the problem is nonlinear. The process that the fixed source equation calculations are embedded in the fitting procedure to update the background cross-sections is very complicated and time-consuming. This problem can be overcome by making the table of background cross-sections with respect to the subgroup levels covering the range from $10^{-4}$ barn to $10^{5}$ barn. Then the subgroup level dependent background cross-sections are interpolated in the fitting procedure.

The process of generating subgroup parameters based on 1-D plate is as follows:

(1) Chose some 1-D plate problems with different dilution, and calculate them by hyperfine group code to acquire $\sigma_{g, k}^{\text {Ref }}$ in Eq.(9). Such problems should be close to the plate cell in real plate-type fuel assemblies to be calculated. The established 1-D plate problems can be divided into several regions including moderator, cladding and fuel. By varying the density of fuel or moderator and area of moderator region, 1-D plate problems with different dilution can be acquired.

(2) Making the interpolation tables or functions of background cross-sections and subgroup levels. Dozens of points of subgroup levels are selected by geometric series from $10^{-4}$ barn to $10^{5}$ barn. After performing the MOC transport calculation to solve Eq.(2) and using Eq.(3), we get the relationship between background cross-sections and subgroup levels.

(3) The least-square problem Eq.(9) is solved to obtain subgroup parameters.

\subsection{Treatment of resonance interference}

We apply the resonance interference factors to deal with the resonance interference effect, because it is easy to implement in subgroup method and accurate to capture the spectrum interference of different isotopes.

The resonance interference factor(RIF) is defined as the effective cross-section of the isotope considered with all other resonance isotopes mixed, divided by the one without any other resonance isotopes. Eq.(10) is used to correct the effective cross-section.

$$
\sigma_{x, k, g}^{\text {eff }}=\sigma_{x, k, g}^{\text {singlehet }} R I F_{x, k, g}=\sigma_{x, k, g}^{\text {single,het }} \frac{\sigma_{x, k, g}^{\text {all }}}{\sigma_{x, k, g}^{\text {single }}}
$$


$\sigma_{x, k, g}^{\text {singlethet }}$ is the effective cross-section before correction which is obtained by the subgroup resonance calculation with the assumption that it is the only resonance isotope in the mixture, and others are all nonresonance ones. During the subgroup calculation, the absorption cross-section of the hypothetical nonresonance isotopes is set to be zero, and the scattering cross-section is forced equal to the potential crosssection as follows:

$$
\begin{gathered}
\sigma_{a, g, k}=0 \\
\sigma_{s, g, k}=\sigma_{p, k}
\end{gathered}
$$

RIF is acquired by calculating two equivalent homogenous problems using continuous energy Monte Carlo codes or hyperfine group codes. The first equivalent homogenous problem contains all resonance isotopes and one scattering isotope. The atomic weight of the scattering isotope equals to that of $\mathrm{H}-1$, and macroscopic scattering cross-section is:

$$
\Sigma_{s, g}=\sigma_{0, g} N_{m}-\sum_{k^{\prime} \neq m} \sigma_{p, k^{\prime}} N_{k^{\prime}}
$$

$N_{m}$ is the density of isotope $m . \sigma_{p, k^{\prime}}$ is the potential scattering cross-section of other resonance isotopes. $\sigma_{0, g}$ is defined as:

$$
\sigma_{0, g}=\frac{\left(\sigma_{a, m, g}+\lambda_{m} \sigma_{p, m}\right) \phi_{g, m}-\lambda_{m} \sigma_{p, m}}{1-\phi_{g, m}}
$$

$\sigma_{a, m, g}$ is the effective absorption cross-section of isotope $m$ from subgroup calculation. $\phi_{g, m}$ is the scalar flux which is acquired by accumulating all subgroup fluxes.

The second equivalent homogenous problem can be established like the first one. Due to no other resonance isotopes contained, Eq.(13) can be rewritten as:

$$
\Sigma_{s, g}=\sigma_{0, g} N_{m}
$$

The equivalent homogenous problems are solved for each resonance isotope and each region. When the fuel is in deep depletion, many resonance isotopes greatly increase the calculations. To overcome the problem, several important resonance isotopes are selected according to their importance. The importance of resonance isotopes is defined as:

$$
I_{k}=N_{k} \sum_{g} \frac{R I_{t, \infty, k, g}}{R I_{t, \min , k, \mathrm{~g}}}
$$

where $R I_{t, \infty, k, g}$ is the resonance integration of isotope $k$ in group $g$ at infinite dilution. It is the maximum value in resonance integral tables. $R I_{t, \min , k, g}$ is the minimum value in resonance integral tables. $R I_{t, \infty, k, g}$ divided by $R I_{t, \text { min, }, \mathrm{g}, \mathrm{g}}$ can represent the extent of resonance effect of the group. 
The subgroup calculation and RIF calculation are only done for important resonance isotopes, other isotopes are all regarded as non-resonance ones. After that, the important resonance isotope with the least importance is selected. When we construct the first equivalent homogenous problem mentioned, all nonimportant resonance isotopes are added to the mixture. The cross-sections obtained from the equivalent homogenous problem with all resonance isotopes are assumed to be the effective self-shielded crosssection of the non-important resonance isotopes. See as follows:

$$
\sigma_{x, k, g}^{\text {eff }} \approx \sigma_{x, k, g}^{\text {all,hom }}
$$

\section{NUMERICAL RESULTS}

JRR-3M is a research reactor loading plate-type fuel. The standard assembly and the followed assembly of the core are shown as Figure 1. The detailed compositions of the assembly are given in Table I. Temperature is $300 \mathrm{~K}$. Detailed geometry information is in reference [7].

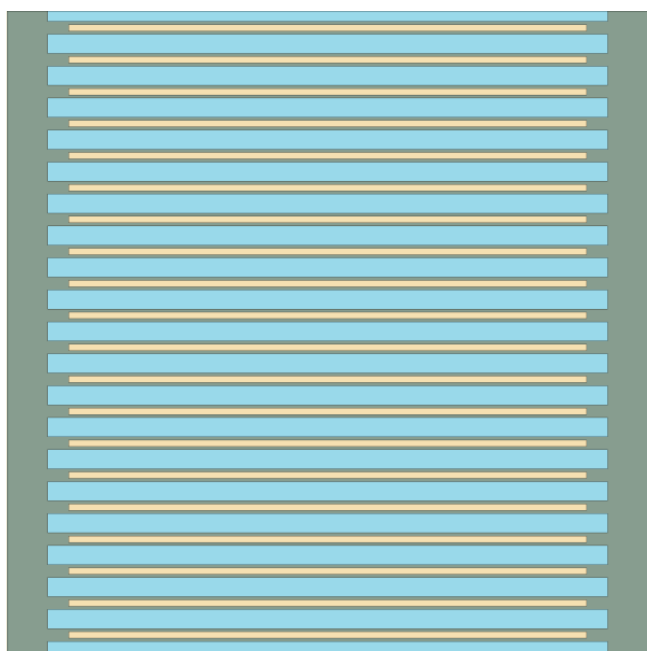

(a) Standard assembly

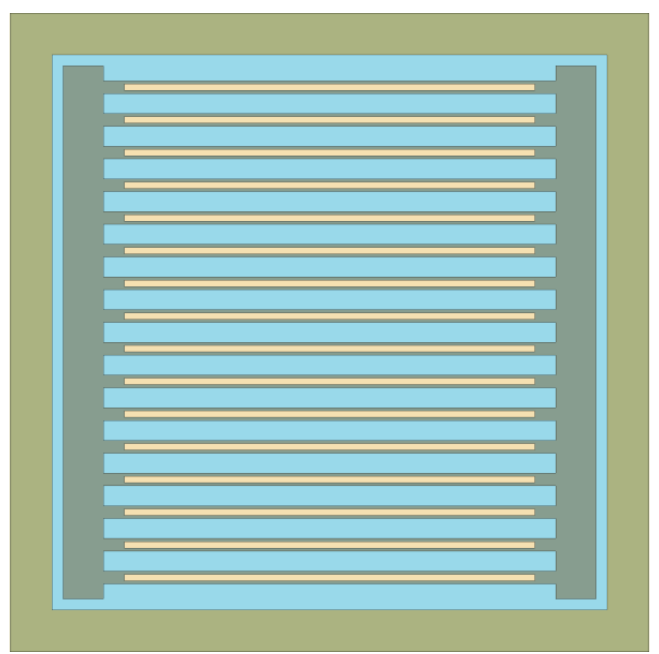

(b) Followed assembly Figure 1. Configurations of JRR-3M fuel assemblies

Table I. Atomic density of the JRR-3M fuel assembly

\begin{tabular}{|c|c|c|}
\hline Component & Isotope & Density $($ barn $/ \mathrm{cm})$ \\
\hline \multirow{3}{*}{ Fuel meat } & U-238 & $6.09033 \mathrm{E}-3$ \\
\cline { 2 - 3 } & U-235 & $1.51804 \mathrm{E}-3$ \\
\cline { 2 - 3 } & Al-27 & $4.13989 \mathrm{E}-2$ \\
\cline { 2 - 3 } & Si-29 & $5.07225 \mathrm{E}-3$ \\
\hline Cladding & Al-27 & $6.00385 \mathrm{E}-2$ \\
\hline \multirow{2}{*}{ Coolant } & H-1 & $6.67656 \mathrm{E}-2$ \\
\cline { 2 - 3 } & O-16 & $3.33828 \mathrm{E}-2$ \\
\hline
\end{tabular}

The PWR lattice calculation code Bamboo-Lattice [9] is improved to do the calculations. In the calculations, the ENDF/B VII.0 evaluated library and the WIMS-D 69-groups structure is adopted, and the resonance groups are from the 15th to 27 th group. The reference results are calculated by the continuous energy Monte Carlo code OpenMC [10]. 
The k-eff results of the problems are shown in Table II, and the error is $88 \mathrm{pcm}$ for standard assembly and $102 \mathrm{pcm}$ for followed assembly. The cross-sections of U-238 and U-235 are also compared with the reference results. The relative errors of standard assembly are generally less than $1 \%$ as shown in Figure 2. The relative errors of followed assembly are a little larger as shown in Figure 3, such as the crosssections of U-238 in group 23 and group 25 nearly $1.5 \%$.

Table II. Comparison of k-eff of fresh fuel assemblies

\begin{tabular}{|c|c|c|c|}
\hline k-eff & Bamboo-lattice & OpenMC & Bias/pcm \\
\hline Standard Assembly & 1.65025 & 1.64937 & +88 \\
\hline Followed Assembly & 1.57736 & 1.57634 & +102 \\
\hline
\end{tabular}

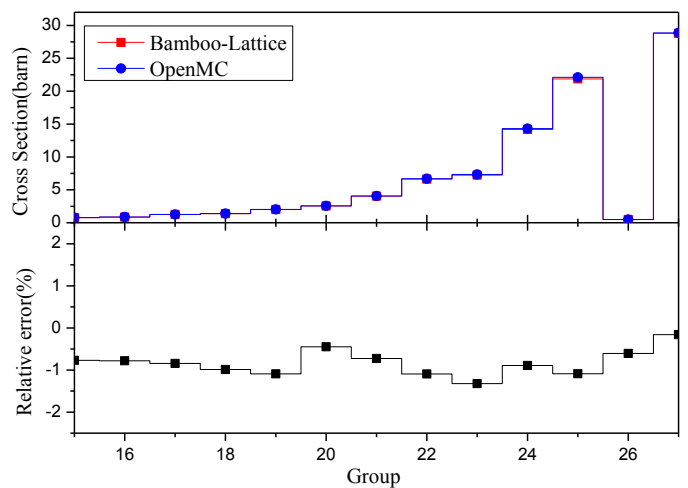

(a) U-238

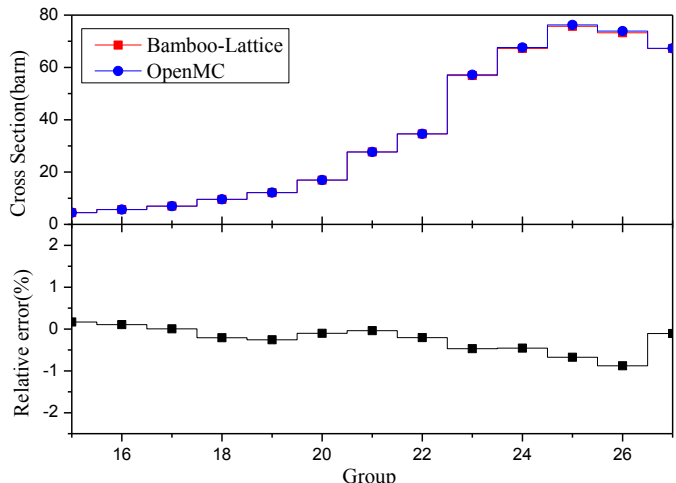

(b) U-235

Figure 2. Comparison of the resonance absorption cross-sections for Standard Assembly

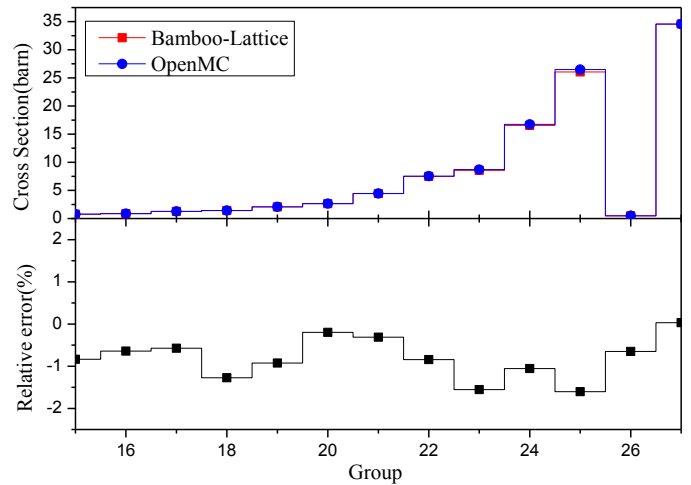

(a) U-238

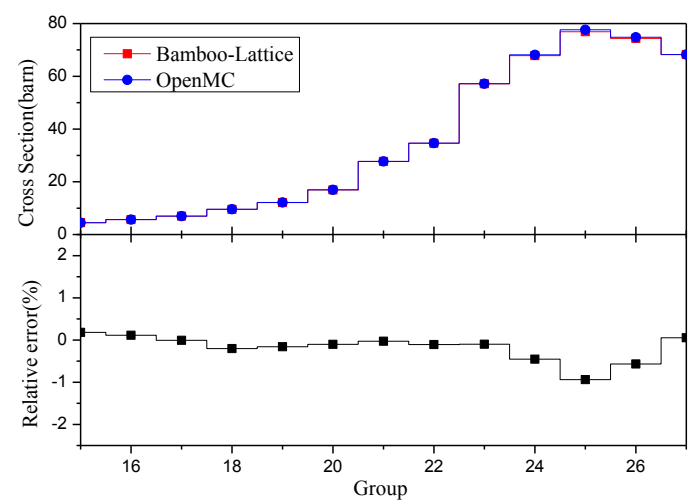

(b) U-235

Figure 3. Comparison of the resonance absorption cross-sections for Followed Assembly

To further test the problems, we calculate these two kinds of assemblies with burnup. To compare the results with reference Monte Carlo results, the isotope composition of the fuel at $30 \mathrm{GWd} / \mathrm{tU}$ is set same in both Bamboo-Lattice and OpenMC. The k-eff results of the problems are shown in Table III. The error is not larger than $150 \mathrm{pcm}$. In the problems, there are many resonance isotopes, but only 5 of them are chosen to be the important resonance isotopes. In this paper, the cross-sections of two important isotopes U-238 and Pu-239 are compared. The relative errors of standard assembly are shown in Figure 4, and that 
of followed assembly are shown in Figure 5. It shows good results for U-238. But for Pu-239 the largest error appears in group $27,2 \%$ for standard assembly and $3.5 \%$ for followed assembly.

Table III. Comparison of k-eff of fuel assemblies with burnup

\begin{tabular}{|c|c|c|c|}
\hline k-eff & Bamboo-lattice & OpenMC & Bias/pcm \\
\hline Standard Assembly & 1.53201 & 1.53094 & +107 \\
\hline Followed Assembly & 1.46085 & 1.45939 & +146 \\
\hline
\end{tabular}

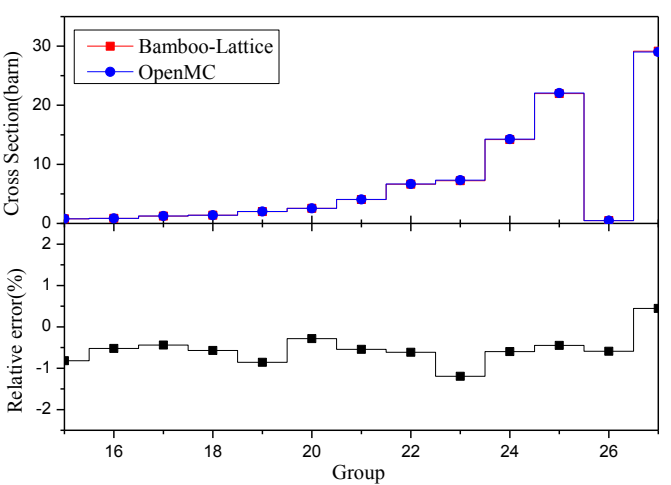

(a) U-238

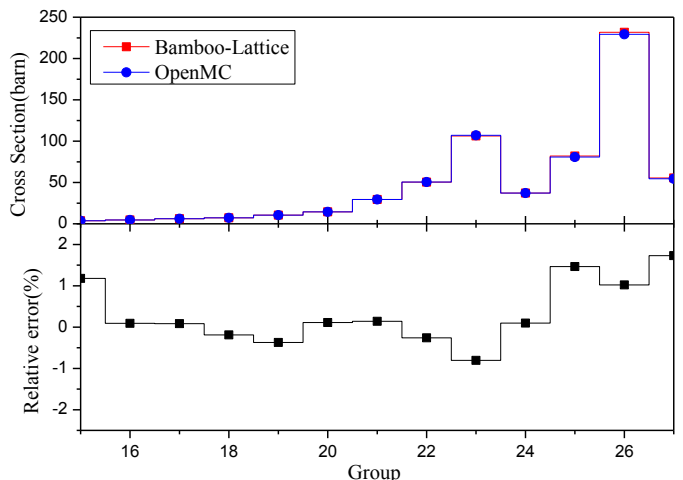

(b) Pu-239

Figure 4. Comparison of the resonance absorption cross-sections for Standard Assembly with burnup

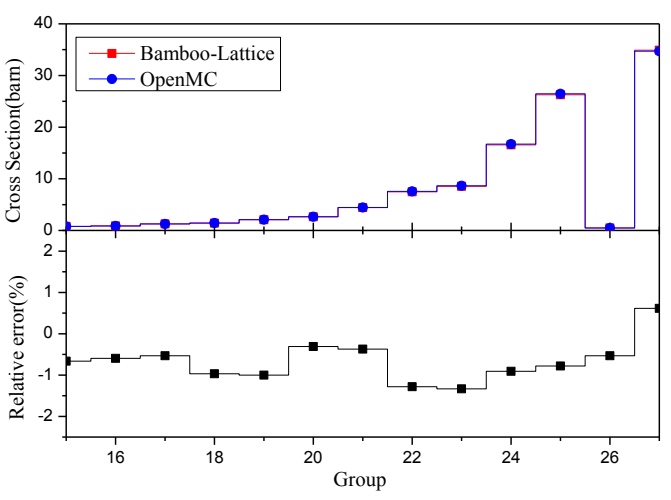

(a) U-238

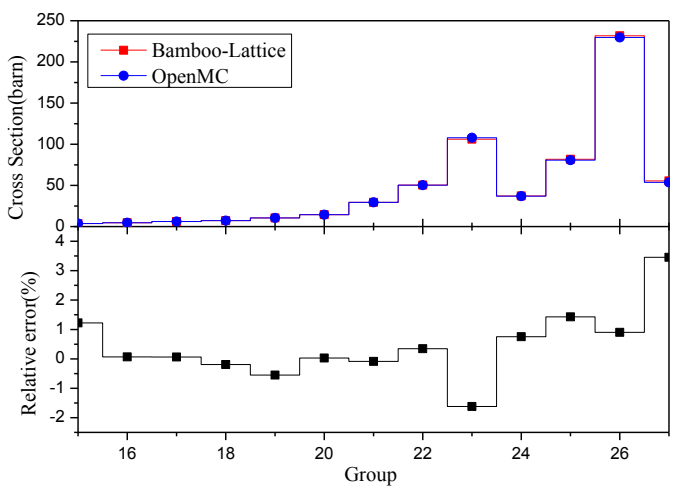

(b) Pu-239

Figure 5. Comparison of the resonance absorption cross-sections for Followed Assembly with burnup

\section{CONCLUSIONS}

In this paper, a resonance calculation scheme is proposed for plate-type fuel assemblies. The subgroup method is good at dealing with complex geometry but inaccurate for plate-type fuel assemblies. To overcome this problem, the subgroup parameters are generated based on heterogeneous problems, 1-D plate. Such 1-D plate should be close to the plate cell in real plate-type assemblies to be calculated. 
The resonance interference effect is also very intense in plate-type assemblies due to their high fuel enrichment. The resonance interference factors generated by equivalent homogenous problems are used to correct the cross-sections obtained by subgroup calculation. To decrease the computational burden, the importance of resonance isotopes is used to classify important ones and non-important ones which are handled by different ways respectively.

The calculation scheme is tested on JRR-3M standard and followed assemblies, and fine results are obtained. The error of $\mathrm{k}$-eff is less than $150 \mathrm{pcm}$ compared with Monte Carlo reference results, and relative errors of cross-sections are generally less than $1.5 \%$, except for some groups of $\mathrm{Pu}-239$.

\section{ACKNOWLEDGMENTS}

This work is financially supported by the National Natural Science Foundation of China (11775171).

\section{REFERENCES}

1. Cullen DE. "Application of The Probability Table Method to Multigroup Calculations of Neutron Transport". Nuclear science and engineering, 55 (4): 387-400(1974).

2. Kim KS, Lee CC, Chang MH, et al. "Monte Carlo resonance treatment for the deterministic transport lattice codes". Nuclear Engineering and Technology, 35(6): 581-595(2003).

3. Liu Y, Martin W, Williams M, et al. "A full-core resonance self-shielding method using a continuousenergy quasi-one-dimensional slowing-down solution that accounts for temperature-dependent fuel subregions and resonance interference". Nuclear Science and Engineering, 180: 247-272(2015).

4. Stamm'ler RJJ. "HELIOS methods". Studsvik Scandpower (2003).

5. Joo HG, Kim GY, Pogosbekyan L. "Subgroup weight generation based on shielded pin-cell cross section conservation". Annals of Nuclear Energy, 36(7): 859-868(2009).

6. Williams M. "Correction of multigroup cross sections for resolved resonance interference in mixed absorbers". Nuclear Science and Engineering, 83: 37-49(1983).

7. Tsuruta H, Ichikawa H, Iwasaki J. "Neutronics design of upgraded JRR-3 research reactor". Japan Atomic Energy Research Inst (1984).

8. $\mathrm{Zu} \mathrm{T}, \mathrm{Xia} \mathrm{F}, \mathrm{Wu} \mathrm{H}$. "An improved fitting method for subgroup parameters based on the heterogeneous cells". Journal of Nuclear Science and Technology, 56(2): 179-192(2019).

9. Li Y, Zhang B, He Q, et al. "Development and verification of PWR-core fuel management calculation code system NECP-Bamboo: Part I Bamboo-Lattice". Nuclear Engineering and Design, 335: 432440(2018).

10. Romano PK, Horelik NE, Herman BR, et al. "OpenMC: A state-of-the-art Monte Carlo code for research and development". Annals of Nuclear Energy, 82: 90-97(2015). 